\title{
Measurement Uncertainty of Dew-Point Temperature in a Two-Pressure Humidity Generator
}

\author{
L. Lages Martins • A. Silva Ribeiro • \\ J. Alves e Sousa · Alistair B. Forbes
}

Received: 8 March 2010 / Accepted: 12 May 2011

(C) Springer Science+Business Media, LLC 2011

\begin{abstract}
This article describes the measurement uncertainty evaluation of the dewpoint temperature when using a two-pressure humidity generator as a reference standard. The estimation of the dew-point temperature involves the solution of a non-linear equation for which iterative solution techniques, such as the Newton-Raphson method, are required. Previous studies have already been carried out using the GUM method and the Monte Carlo method but have not discussed the impact of the approximate numerical method used to provide the temperature estimation. One of the aims of this article is to take this approximation into account. Following the guidelines presented in the GUM Supplement 1, two alternative approaches can be developed: the forward measurement uncertainty propagation by the Monte Carlo method when using the Newton-Raphson numerical procedure; and the inverse measurement uncertainty propagation by Bayesian inference, based on prior available information regarding the usual dispersion of values obtained by the calibration process. The measurement uncertainties obtained using these two methods can be compared with previous results. Other relevant issues concerning this research are the broad application to measurements that require hygrometric conditions obtained from two-pressure humidity generators and, also, the ability to provide a solution that can be applied to similar iterative models. The research also studied the factors influencing both the use of the Monte Carlo method (such as the seed value and the convergence parameter) and the inverse
\end{abstract}

L. L. Martins $(\varangle) \cdot$ A. S. Ribeiro

Laboratório Nacional de Engenharia Civil (LNEC), Lisbon, Portugal

e-mail: 1fmartins@lnec.pt

J. Alves e Sousa

Laboratório Regional de Engenharia Civil (LREC), Funchal, Portugal

A. B. Forbes

National Physical Laboratory (NPL), Teddington, UK

Published online: 31 May 2011 
uncertainty propagation using Bayesian inference (such as the pre-assigned tolerance, prior estimate, and standard deviation) in terms of their accuracy and adequacy.

Keywords Dew-point temperature $\cdot$ Iterative model $\cdot$ Measurement uncertainty

\section{Introduction}

The measurement of the dew-point temperature can be made either directly by a condensation hygrometer or indirectly using hygrometric instrumentation that measures input quantities such as the temperature and pressure and uses explicit mathematical models. Traceability is provided by the calibration process, establishing the link to the SI and characterizing the instrumentation accuracy level through the stated measurement uncertainty.

Metrology laboratories can provide the connection between the top levels - national metrology institutes (NMIs) and the BIPM-and lower level laboratories in industry and, therefore, have an important role in the establishment of the hygrometric traceability chains. In some cases, highly accurate reference standards are already available to be used by secondary metrology laboratories that are able to generate physical conditions according to the primary definition of the relevant quantities with low measurement uncertainties, as in the case of hygrometric instrumentation.

Some of these standards, however, are based on complex mathematical models, and require that the method applied to the evaluation of measurement uncertainty should be fit to purpose. Knowing that the international framework established by the publication of the GUM [1] in 1995 is especially suited to linear models while its Supplement 1 [2] is more able to adequately handle non-linear models, the issue of the choice of methodology is relevant for metrologists.

Nowadays, humidity generators are becoming important reference hygrometric standards in many metrology laboratories as they provide stable and uniform test conditions. In the case of a two-pressure humidity generator, the dew-point measurement is obtained indirectly from pressure and temperature measurements and an iterative mathematical model. The particular nature of this model constitutes a challenge in terms of measurement uncertainty evaluation.

Some authors, such as [3], have adopted the GUM method to obtain a solution for this specific problem. An alternative Monte Carlo approach has been proposed [4] as more suitable to deal with the non-linearity of the mathematical model. The results obtained from both approaches are very close and show only minor differences. However, the above-mentioned Monte Carlo approach [4] involves a simplified numerical method for the determination of the dew-point temperature and does not take into account the uncertainty related to the saturator efficiency. In this study, this influence factor is included in the evaluation.

The two alternative approaches proposed in this study were based on GUM Supplement 1 [2]: forward measurement uncertainty propagation by the Monte Carlo method $(\mathrm{MCM})$ and the inverse measurement uncertainty propagation method by Bayesian inference $(\mathrm{BI})$. 